\title{
Effect of the Temperature on chromium precipitation in faucets
}

\author{
Huang Tingting ${ }^{1,2,3}$ \\ ${ }^{1}$ Nanan Testing Institute of Quality and Metrology, Quanzhou, Fujian, China \\ ${ }^{2}$ National Quality Supervision and Inspection Center of Plumbing \& Sanitary Ware (Fujian), Quanzhou, Fujian, China \\ ${ }^{3}$ Nanan Market Supervision and Comprehensive Law Enforcement Brigade, Quanzhou, Fujian, China
}

\begin{abstract}
The research was studying the chromium precipitation in faucets which were extracted at ambient temperature of $20{ }^{\circ} \mathrm{C}, 40{ }^{\circ} \mathrm{C}$ and $60{ }^{\circ} \mathrm{C}$ by extraction solution, the results showed that in general, the chromium extraction in the faucets increased as the temperature rises. And the chromium extraction in different batches and different samples in the same batch varied greatly, mainly affected by materials and the processing technology. After further analysis, the detection showed that $\mathrm{Cr}$ VI in chromium precipitation was between $83 \%$ and $103 \%$ of the total chromium content. This indicated that Cr VI was the main form of chromium in extraction solution, which will be harmful to human health.
\end{abstract}

\section{Introduction}

Everyone's daily life is inseparable from water, water quality, especially drinking water quality is closely related to human health. As the endpoint devices of drinking water system, the precipitation of harmful contaminants from faucets directly affect the quality of water and the safety of residents' health. Most of the faucets in the electroplating process, often use chromate and dichromate as electroplating reagents, and most of the faucets material also have trace amounts of chromium elements, so the extraction of chromium in the faucets is inevitable. The precipitated chromium mainly exists in the form of of $\mathrm{Cr}$ III and Cr VI, of which Cr VI is a carcinogen, and is easily absorbed by many tissues and organs in the human body and accumulated, which will harm human health [1].

According to the research, in the daily use of room temperature, the small range of $\mathrm{pH}$ and electrical conductivity had little influence on the chromium precipitation, the influence factor is the extraction time [2]. With the development of science and technology and the improvement of living standards, now the general household faucets have a temperature control function. The precipitation of harmful contaminants from faucets is a complex electrochemical corrosion process, which is also affected by the temperature [3]. This research mainly studied the extraction of chromium in faucets in different temperature conditions and analysis the main form of chromium, aiming at providing scientific basis for the safety of drinking water for residents and industrial production.

\section{Experiment}

\subsection{Instruments and Reagents}

\subsubsection{Instruments}

Nexion $^{\mathrm{TM}}$ 350X ICPMS (USA Perkin Elmer company), UV spectrophotometer (UV 2600, SHIMADZU), Mini-q Reference Ultra Pure Water system (USA Millipore company), PH meter ( Mettler-toledo), Conductivity meter (Mettler -toledo).

\subsubsection{Reagents}

Standard solution:1 g/L chromium (GBW08614) was purchased from China Institute of Metrology, $1 \mathrm{~g} / \mathrm{L} \mathrm{Cr} \mathrm{VI}$ (BW30030-100-N-50) was purchased from Beijing Tanmo.

Other reagents: waterless $\mathrm{NaHCO}_{3}(\mathrm{AR}), 5 \%$ sodium hypochlorite solution (AR), nitric acid (GR) were all purchased from Shanghai Guo pharmaceutical group chemical reagents Co. Ltd.; Ultra Pure Water (electrical resistivity $\geq 18.2 \mathrm{M} \Omega \cdot \mathrm{cm}$ ).

\subsection{Samples extraction}

According to the GB18145-2014 Appendix B method [4], the extraction solution that simulates tap water is formulated. In order to carry on the test better, this experiment chose the inferior faucets as samples, a total of 5 batches, respectively, 1\#, 2\#, 3\#, 4\#, 5\#. Each batch had 9 parallel samples. The samples were rinsed with tap water for 15 min,then 3 times with ultra pure water, the residue and dirt from the sample were washed out, and 
then 3 times with extraction solution. Each batch of samples was taken in 3 parallel samples at an ambient temperature of $20{ }^{\circ} \mathrm{C}, 40{ }^{\circ} \mathrm{C}, 60{ }^{\circ} \mathrm{C}$ for extraction experiments. The samples were fully filled with extraction solution, and the sample handle switch was in a fully open state, and both ends of the sample were tightly plugged with a clean rubber plug covered with Teflon film. After the sample filled with the extraction solution had stabilized at its respective ambient temperature of no more than $72 \mathrm{~h}$, the extraction solution in the sample was replaced in accordance with appendix B of GB181452014, and the held for $16 \mathrm{~h}$ extraction solution in the sample was collected for 5 days after stabilization. The $3 \#$ samples were repeated the above steps and collected the extraction solution for the next 5 days. The $3 \#$ samples originally incubated at $20{ }^{\circ} \mathrm{C}$ were then exchanged with the $60{ }^{\circ} \mathrm{C}$ samples, and the extraction solution is collected for the next 5 days. The collected extraction fluid was divided into two parts, one with thick nitric acid to make the solution $\mathrm{pH}<2$, placed at room temperature of more than $24 \mathrm{~h}$ to ensure that the metal was completely dissolved prior to analysis, was used to measure the total chromium, and the other was for the detection of $\mathrm{Cr} \mathrm{VI}$.

\subsection{Test method}

\subsubsection{ICP-MS}

Following consideration of the factors of abundance, interference and sensitivity, selected ${ }^{52} \mathrm{Cr}$ isotope for testing, ${ }^{45} \mathrm{Sc}$ as the internal standard element, using the method of online marking.
Instrument working conditions: RF (1.6 KW), Carrier Gas (Argon): $0.90 \mathrm{~L} / \mathrm{min}$, Cooling gas (Argon): 15.0 L/min, Helium: $4.5 \mathrm{~mL} / \mathrm{min}$, Sweeps/reading (20), Readings/replicate (2), Number of Replicate (2), Detector Mode (Dual), KED mode.

\subsubsection{UV spectrophotometer}

Cr VI in extraction solution was detected according to GB/T 5750.6-2006: Diphenylcarbonyl dihydrazine spectrophotometric method [5], when the detected concentration exceeded the upper limit of the standard curve, the dilution and re-determination was made with fresh preparation extraction solution.

\section{Results and discussion}

\subsection{Chromium precipitation in the faucets affected by temperature}

The experiment selected a total of 5 batches, 9 parallel samples per batch, and 3 parallel samples at three degree ambient temperature. The concentration of total chromium of 5 days of extraction solution were detected by ICP-MS. Each batch of 3 parallel samples per day for the weighted average was defined as the derived value of the batch. The sample total chromium precipitation concentrations changed with temperature rises were shown in Figure 1.

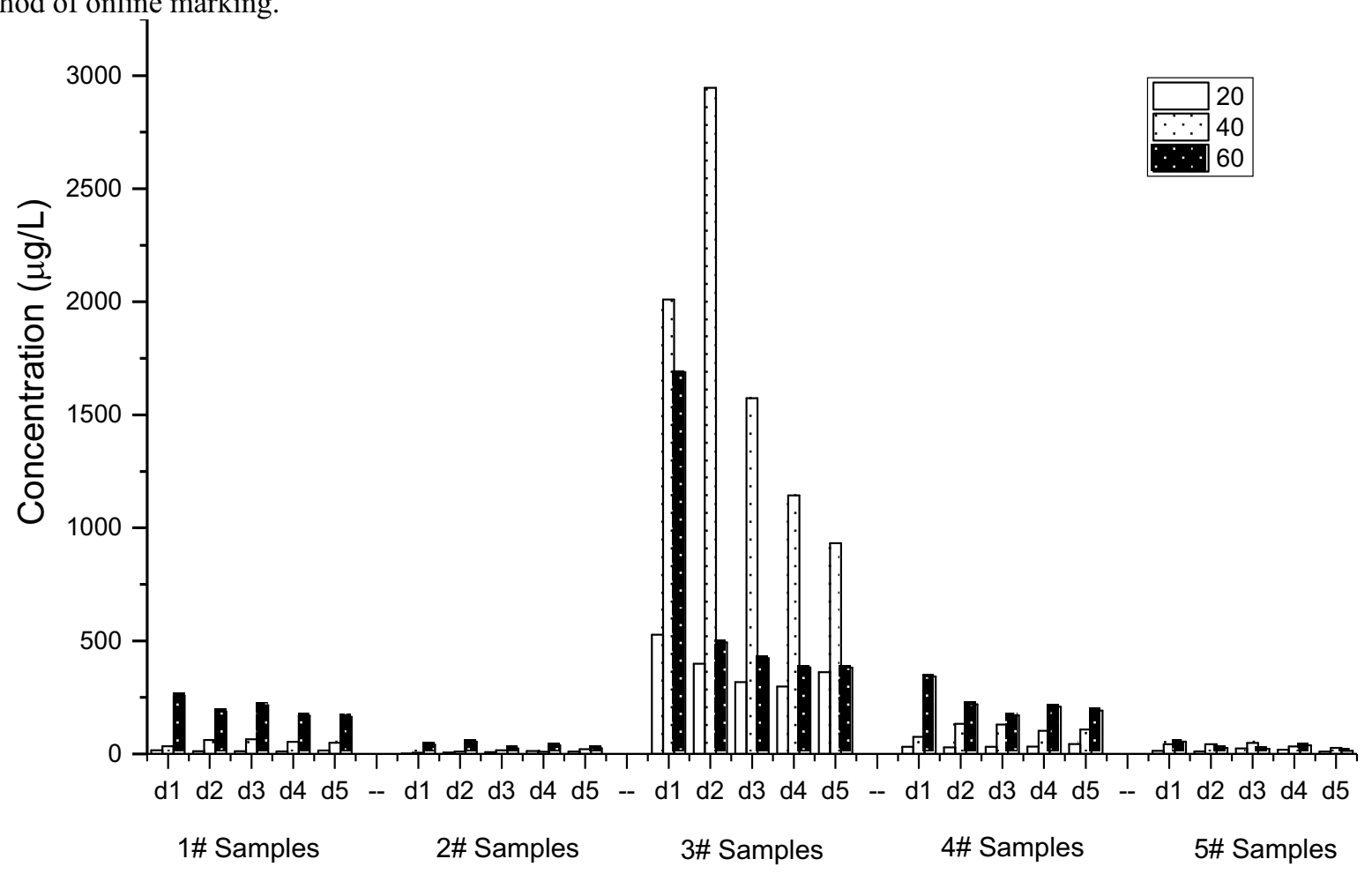

Figure 1. Total chromium precipitation in 5 batches samples for 5 days at $20{ }^{\circ} \mathrm{C}, 40{ }^{\circ} \mathrm{C}, 60{ }^{\circ} \mathrm{C}$ 


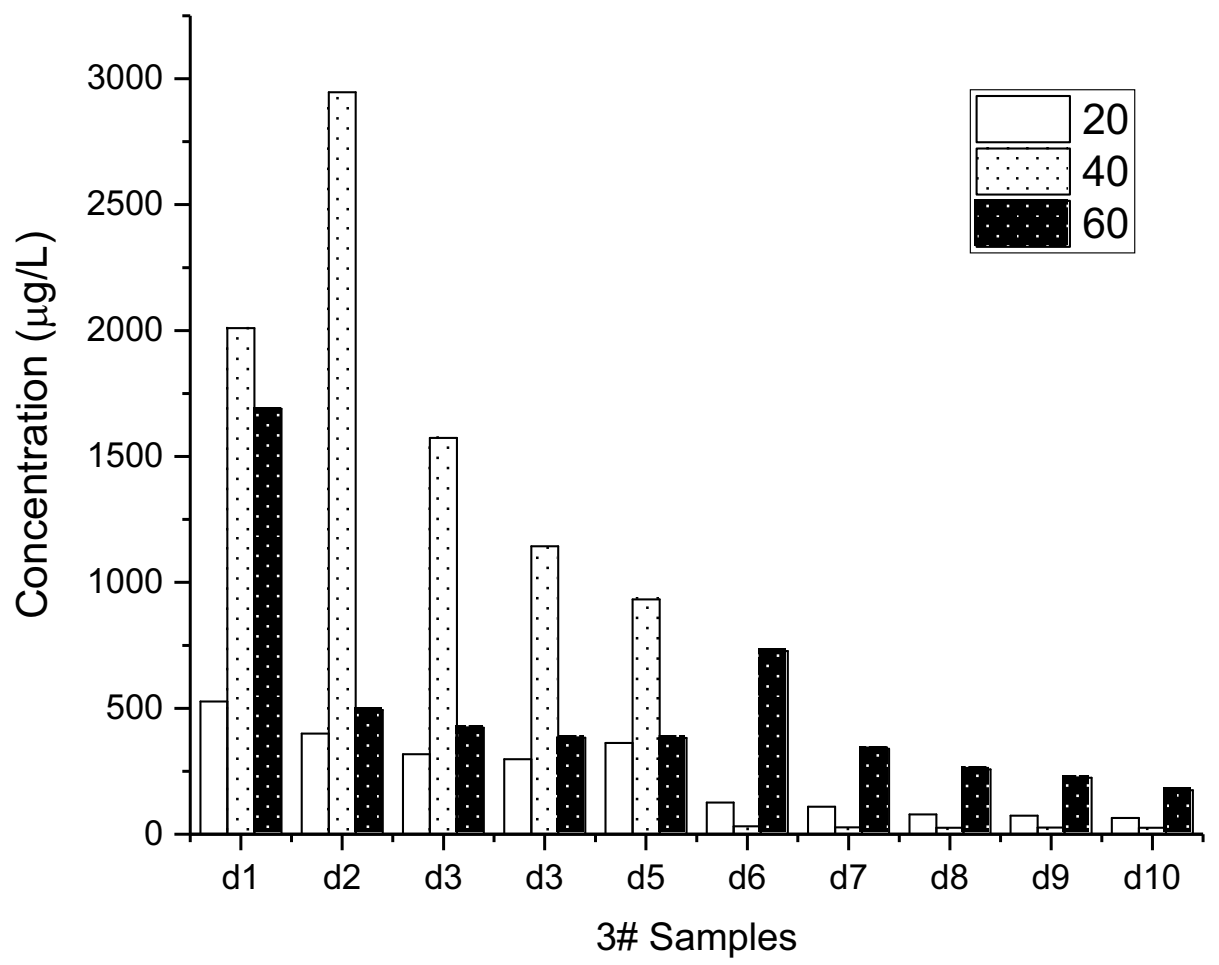

Figure 2. Total Chromium Precipitation in $3 \#$ samples extracted for 10 days

Figure 1 showed that the chromium precipitation in the 5 batches samples varied widely, especially the $3 \#$ samples were very high. Except for the $3 \#$ samples, the chromium precipitation of other samples generally increased with the rise of temperature, and the chromium precipitation did not show a regular and obvious reduction with the increase of extracting days. The concentration of chromium precipitation of the $3 \#$ samples showed a trend of $40{ }^{\circ} \mathrm{C}>60{ }^{\circ} \mathrm{C}>20{ }^{\circ} \mathrm{C}$, and the precipitation had an obvious trend of decreasing with the increase of extracting days.

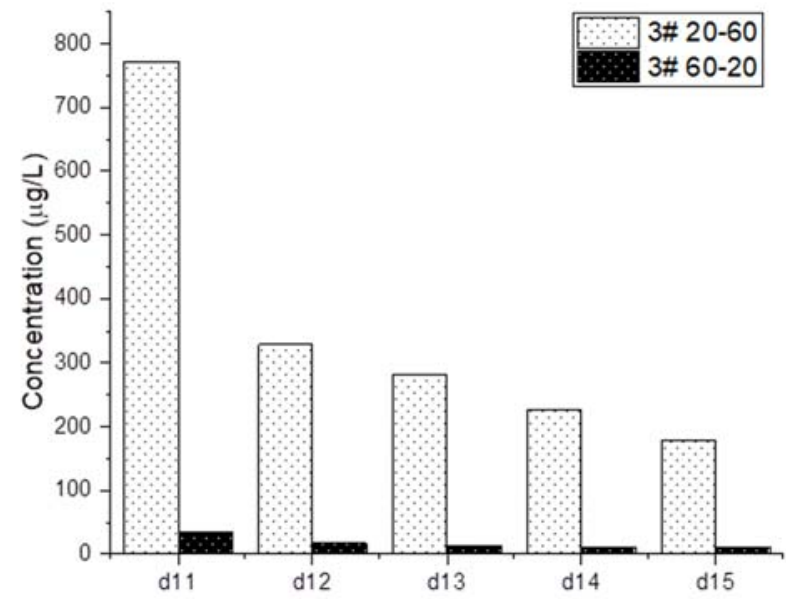

Figure 3. Total chromium precipitation of $3 \#$ sample after $20^{\circ} \mathrm{C}$ and $60^{\circ} \mathrm{C}$ extraction temperature replacement

In order to further study the total chromium precipitation of the sample 3\#, the 5-day extraction solution of the next cycle was continuously collected and tested, as shown in Figure 2. The figure showed that in the second round of immersion extraction, the total chromium precipitation was $60{ }^{\circ} \mathrm{C}>20{ }^{\circ} \mathrm{C}>40{ }^{\circ} \mathrm{C}$, among which $40{ }^{\circ} \mathrm{C}$ showed a sharp decrease. For further investigated the effect of temperature on the precipitation of total chromium in faucets $3 \#$ samples which incubated at $20{ }^{\circ} \mathrm{C}$ for the first 10 days were selected and placed at $60{ }^{\circ} \mathrm{C}$. The samples extracted at $60{ }^{\circ} \mathrm{C}$ were replaced and incubated at $20{ }^{\circ} \mathrm{C}$ for the third round of extraction for 5 days. The results were shown in Figure 3. The samples (3\#20-60) originally extracted at $20{ }^{\circ} \mathrm{C}$ showed a sharp increase of chromium precipitation at $60{ }^{\circ} \mathrm{C}$, and showed a decreasing trend with the increase of extracting days. However, the chromium precipitation of (3\#60-20) which was originally incubated at $60^{\circ} \mathrm{C}$ was very little at $20^{\circ} \mathrm{C}$, which decreased from $35 \mu \mathrm{g} / \mathrm{L}$ to $10 \mu \mathrm{g} / \mathrm{L}$ as the days gone by.

The above experimental results indicated that the precipitation of chromium in the test samples was greatly affected by temperature, in general, the concentration of chromium precipitation increased at the higher temperature. The abnormal precipitation of $3 \#$ samples in the first 5 days should be affected by the process technology. The general temperature of decorative chromium in the electroplating process of ordinary faucets is $40-45^{\circ} \mathrm{C}$. If the faucets are not cleaned well after electroplating, the residual electroplating reagents should attached to the inner cavity of the faucets are more likely to extracted under suitable temperature conditions. Fig.4,Fig.5 and fig.6 respectively showed the 10-day total chromium precipitation of the three parallel samples of the $3 \#$ sample. The precipitation trends of the three parallel samples were similar, but the concentration of precipitation varied greatly, indicating that the chromium precipitation of individual faucet in the same batch varied greatly also. 


\subsection{Morphology analysis of chromium precipitation in samples}

For further research of the morphology of the chromium which was extracted from faucets samples, the collected extraction solution was tested by Diphenylcarbonic dihydrazine spectrophotometric method. The result showed that Cr VI which was more toxic to human body accounted for $83 \% \sim 103 \%$ of the total chromium precipitation. So it indicated that the extraction solution was harmful to human.

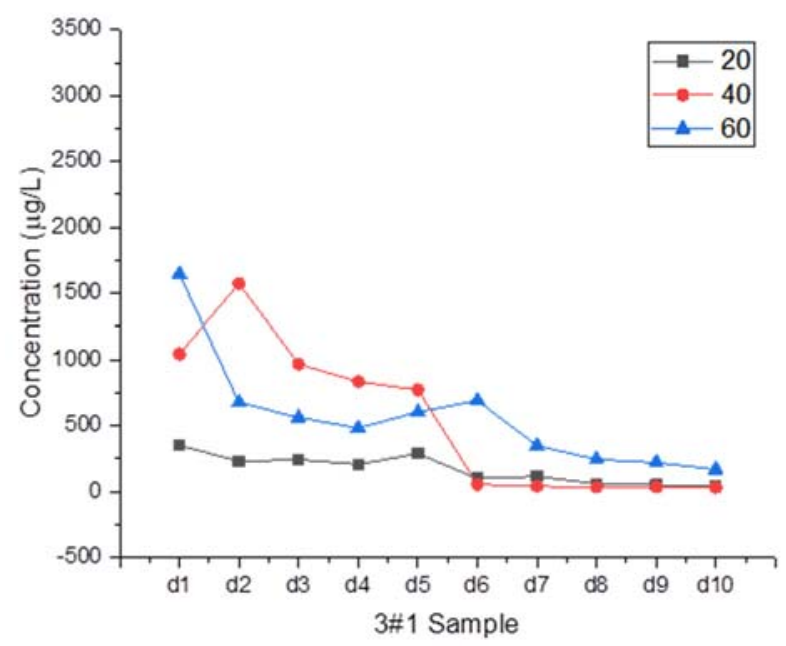

Figure 4. Total Chromium Precipitation of 3\#1sample extracting for 10 Days

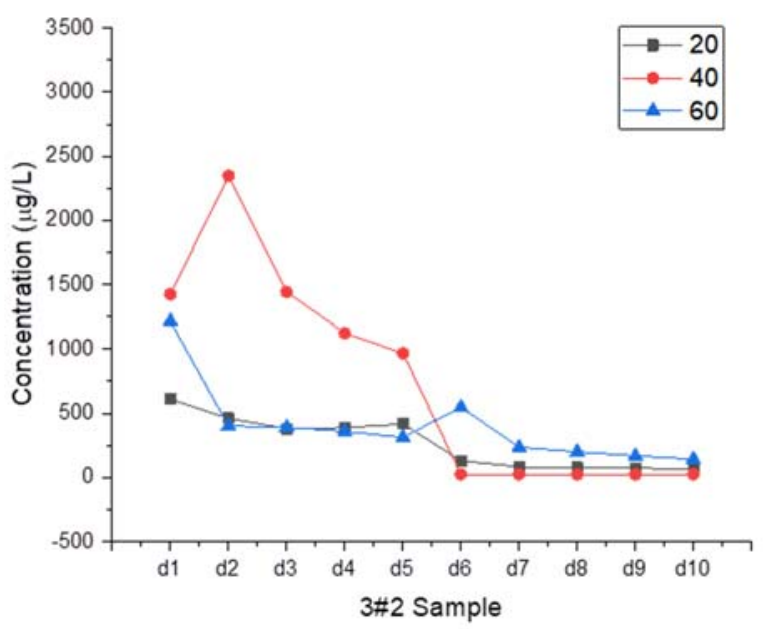

Figure 5. Total Chromium Precipitation of $3 \# 2$ sample extracting for 10 Days

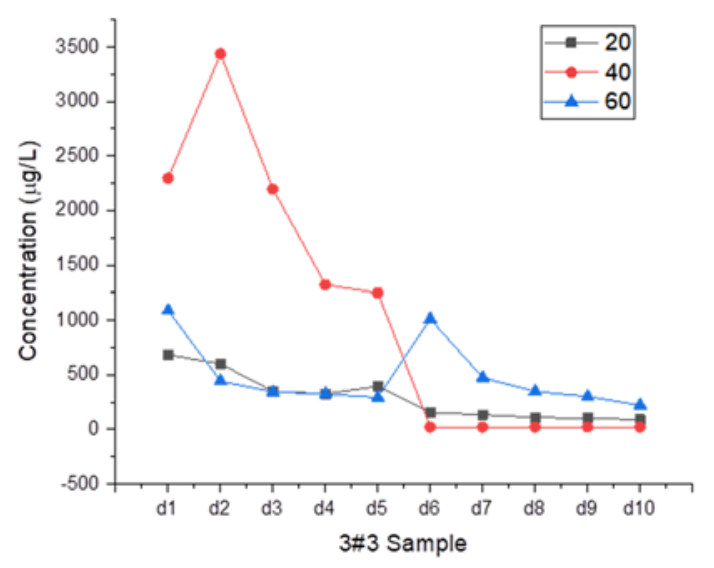

Figure 6. Total Chromium Precipitation of $3 \# 2$ sample extracting for 10 Days

\section{Conclusions}

In this study, through detecting the precipitation of chromium in faucets which were extracted at $20{ }^{\circ} \mathrm{C}$, $40{ }^{\circ} \mathrm{C}, 60{ }^{\circ} \mathrm{C}$ environment temperature, the results showed that under normal circumstances, the chromium precipitation at $60{ }^{\circ} \mathrm{C}$ was the most, $20{ }^{\circ} \mathrm{C}$ was the least, indicating that the chromium precipitation in the faucets increases with the rise of temperature. And the precipitation between different batches and different samples from the same batch were greatly different, which was mainly affected by the faucets material and processing technology. Further detection showed that the precipitation of chromium mainly existed in the form of Cr VI, which will cause harm to human health. Therefore, in daily life, quality and qualified faucets should be selected, and in the process of use, the long-term retention of water in the faucets should be avoided as drinking water (especially under high temperature environment).

\section{Acknowledgments}

This research was supported by Quanzhou Science and Technology Project (Project No. 2018C093R).

\section{References}

1. X. Zeng, Y.G. Huang, J. H.B. Polytech. Univ., 36, 2, 18-21(2020).

2. T.T. Huang, IOP Conf. Ser.: Earth Environ. Sci. 199 032082 (2018).

3. L. Zlatanovic, J.P. Hoek, J.H.G. Vreeburg, Water Res., 123: 761-72 (2017).

4. GB 18145-2014, Ceramic cartridge faucets[S].

5. GB 5750.6-2006, Standard examination methods for drinking water-Metal parameters[S]. 